\title{
Water vapor differential absorption lidar measurements using a diode-pumped all-solid-state laser at $935 \mathrm{~nm}$
}

\author{
A. Fix · G. Ehret · J. Löhring · D. Hoffmann · M. Alpers
}

Received: 10 May 2010 / Revised version: 12 August 2010 / Published online: 24 November 2010

(C) The Author(s) 2010. This article is published with open access at Springerlink.com

\begin{abstract}
A diode-pumped, single-frequency laser system emitting at $935 \mathrm{~nm}$ has recently been developed to serve as the transmitter for water vapor differential absorption lidar (DIAL) measurements. This laser uses Nd:YGG $\left(\mathrm{Y}_{3} \mathrm{Ga}_{5} \mathrm{O}_{12}\right)$ as the active medium and emits radiation directly at $935 \mathrm{~nm}$ without the need of additional frequency conversion processes. The system was diode-pumped at $806 \mathrm{~nm}$ and was built up in a master-oscillator-poweramplifier configuration. It generates more than $30 \mathrm{~mJ}$ of pulse energy at $100 \mathrm{~Hz}$ repetition rate with a beam quality $\left(M^{2}\right)$ of better than 1.4. Since water vapor DIAL demands for stringent requirements of the spectral properties those were carefully investigated in the scope of this paper. Single-frequency operation is achieved by injection seeding and active length control of the oscillator cavity. The range of continuously tunable single-frequency radiation extends
\end{abstract}

\footnotetext{
A. Fix $(\bowtie) \cdot$ G. Ehret

Institut für Physik der Atmosphäre, Deutsches Zentrum für Luftund Raumfahrt (DLR), 82234 Oberpfaffenhofen, Germany e-mail: Andreas.Fix@dlr.de

G. Ehret

e-mail: Gerhard.Ehret@dlr.de
}

J. Löhring · D. Hoffmann

Fraunhofer-Institut für Lasertechnik (ILT), Steinbachstr. 15, 52074 Aachen, Germany

J. Löhring

e-mail: jens.loehring@ilt.fraunhofer.de

D. Hoffmann

e-mail: hansdieter.hoffmann@ilt.fraunhofer.de

\section{Alpers}

Raumfahrt-Agentur, Deutsches Zentrum für Luft- und Raumfahrt (DLR), Königswinterer Straße 522-524, 53227 Bonn, Germany

e-mail: Matthias.Alpers@dlr.de to $\sim 0.4 \mathrm{~nm}$ centered around $935.31 \mathrm{~nm}$. Values of the spectral purity of $>99.996 \%$ were determined using long-pass absorption measurements in the atmosphere exceeding the requirements by a large margin. Finally, for the first time water vapor DIAL measurements were performed using a Nd:YGG laser. The reported results show much promise of these directly pumped lasers at $935 \mathrm{~nm}$ for future spaceborne but also airborne water vapor lidar systems.

\section{Introduction}

Water vapor $\left(\mathrm{H}_{2} \mathrm{O}\right)$ is the most abundant and important greenhouse gas in the atmosphere [1]. It controls weather and climate on all temporal and spatial scales, is also important for a variety of chemical processes in the atmosphere, and plays a central role in the global energy and water budget.

The knowledge of vertically resolved profiles with high accuracy and precision of atmospheric humidity from the ground throughout the whole troposphere and, particularly, up to the lower stratosphere is therefore a prerequisite for numerical weather prediction (NWP) [2-4] and climate research $[5,6]$. Unfortunately, no global observation system is currently available that fulfills the requirements defined by the World Climate Research Programme (WCRP) of the World Meteorological Organization (WMO).

Since the differential absorption lidar (DIAL) technique applied from satellites shows high potential to meet those requirements [7-9] there is need for an exploratory satellite mission demonstrating high-vertical resolution water vapor profiles by active remote sensing [5]. In recent years several lidar missions have thus been proposed to measure water vapor profiles from space [10-13]. However, the feasibility of a space mission heavily depends on the availabil- 
ity of mature and reliable laser sources at $\sim 935 \mathrm{~nm}$ in the absorption band of $\mathrm{H}_{2} \mathrm{O}$ with high electrical-to-optical efficiency. Although DIAL systems have been successfully deployed on aircraft, their laser transmitters do not quite meet the desired efficiency requirements yet. In most cases either Ti:Sapphire lasers (e.g. [14]) or optical parametric oscillators (OPOs) (e.g. [15]) have been used. In particular, an airborne demonstrator based on an OPO at $935 \mathrm{~nm}$ was developed in recent years and has successfully validated the fourwavelength concept using three different absorption lines to measure the water vapor profile from ground to the lower stratosphere [15]. Those sources are pumped making use of the second harmonic $(532 \mathrm{~nm})$ of the ubiquitous $\mathrm{Nd}$ :YAG laser. In this frequency conversion process, however, about half of the pump energy is wasted.

In principle it should be possible to come up with more efficient and less complex diode-pumped all-solid-state lasers that directly generate wavelengths in the respective wavelength range without using any additional wavelength conversion process. It has been suggested that $\mathrm{Nd}$-doped $\mathrm{Y}_{3} \mathrm{Ga}_{x} \mathrm{Al}_{(5-x)} \mathrm{O}_{12}$ lasers can be specifically tuned by changing the material composition $(0<x<5)$ and thus the lattice parameters to generate wavelengths at $935 \mathrm{~nm}[16,17]$. Therefore this material class shows much prospect to generate the wavelengths required for water vapor DIAL $[8,10,12]$ more efficiently than competing devices such as Ti:Sapphire lasers [14] or OPOs [15] which require pump wavelengths in the green spectral range. Nevertheless, these materials have not yet been investigated to a stage that allows their use in practical lidar systems. Therefore, further studies on this critical technology were strongly advised to support mission feasibility [18].

In a companion paper [19], however, a highly efficient, diode-pumped and single-frequency laser, which uses $\mathrm{Nd}$ :YGG $\left(\mathrm{Y}_{3} \mathrm{Ga}_{5} \mathrm{O}_{12}\right)$ as the active material to directly generate laser radiation at $935 \mathrm{~nm}$ was described for the first time. Here, we investigate whether the properties of this system and, in particular, its spectral properties are adequate to meet the stringent requirement for water vapor DIAL. Emphasis was put on the measurement of the spectral purity using long-pass absorption. Finally, ground-based measurement of the water vapor profile were performed. To the best of our knowledge this is the first report of water vapor DIAL measurements using a diode-pumped laser at $935 \mathrm{~nm}$ without additional frequency conversion.

The paper is structured as follows. At the beginning the experimental set-up is described including a brief review of the relevant properties of the laser system. Subsequently, the spectral properties and in particular the spectral purity is carefully analyzed. The paper concludes with the description of water vapor measurements.

\section{Experimental set-up}

\subsection{Transmitter system}

As the laser transmitter a diode-pumped Nd:YGG (Nd-doped $\mathrm{Y}_{3} \mathrm{Ga}_{5} \mathrm{O}_{12}$ ) master-oscillator-power-amplifier (MOPA) laser was used that has recently been developed [19] to demonstrate its potential for water vapor DIAL measurements particularly from a spaceborne platform. The master-oscillator-power amplifier set-up was described in detail in a companion paper [19]. Here, we only review its main properties that are listed in Table 1.

The Nd:YGG oscillator is set up as a linear cavity in twisted mode configuration with an electro-optical Qswitch. The optics were designed in a way that parasitic lasing at $1062 \mathrm{~nm}$, the gain maximum of Nd:YGG, was prevented. The crystal was a $1 \%$-doped Nd:YGG crystal with a diameter of $3 \mathrm{~mm}$ and a length of $10 \mathrm{~mm}$. It was pumped from two sides by fiber-coupled passively cooled diode bars. At an energy from the fiber-coupled pump diodes of $75 \mathrm{~mJ}$, which was chosen as the working point, the laser generated $4.3 \mathrm{~mJ}$ in Q-switched operation and a pulse duration of $52.5 \mathrm{~ns}$ (FWHM), at a repetition rate of $100 \mathrm{~Hz}$. The $\mathrm{M}^{2}$ value was experimentally determined to be 1.06 . The oscillator beam was mode matched to the astigmatic amplifier mode using two cylindrical telescopes.

In order to boost the pulse energy into the multi-tenmillijoule range an amplifier stage with a hybrid cavity according to the INNOSLAB concept [20] was employed. The thin Nd:YGG slab crystal $\left(14.8 \times 15 \times 2 \mathrm{~mm}^{3}\right)$ was end pumped from both sides by a total of 44 laser diode bars through a free-space transfer optic. At a maximum diode pump energy of $828 \mathrm{~mJ}$ within $200 \mu$ s at the crystal and 13 passes of the oscillator beam through the slab crystal, the MOPA generated $33.2 \mathrm{~mJ}$ of energy. The astigmatism induced by the amplifier design was mostly compensated using cylindrical telescopes. The $M^{2}$-value of the outgoing beam was measured to be less than 1.4 and the divergence in the horizontal and vertical axes was $0.32 \mathrm{mrad}$ and

Table 1 Summary of laser properties in single-longitudinal-mode operation

\begin{tabular}{ll}
\hline Parameter & Value \\
\hline Oscillator pulse energy & $4.3 \mathrm{~mJ}$ \\
MOPA pulse energy & $30.5 \mathrm{~mJ}$ \\
Repetition rate & $100 \mathrm{~Hz}$ \\
Pulse duration (FWHM) & $52.5 \mathrm{~ns}$ \\
Tuning range & $935.10 \mathrm{~nm}-935.55 \mathrm{~nm}$ (vac.) \\
(single-frequency mode) & \\
Spectral width & $<15 \mathrm{MHz}(\mathrm{rms})$ \\
Stability of center wavelength & $<3 \mathrm{MHz}(\mathrm{rms})$ \\
Beam quality $\left(M^{2}\right)$ & $<1.4$ \\
\hline
\end{tabular}


$0.41 \mathrm{mrad}$, respectively which fits well to the field-of-view of the receiving system (see Sect. 2.3).

The energy is well in the range for what is required for ground-based or airborne DIAL systems [8, 9, 21]. For spaceborne applications an energy of $\sim 80 \mathrm{~mJ}$ was suggested $[11,12]$. Further enhancement of the amplifier output energy, however, is possible by adding a subsequent amplifier stage of identical design.

The output spectrum of the free-running MOPA shows two distinct emission lines at $935.31 \mathrm{~nm}$ and $938.43 \mathrm{~nm}$, respectively. A spectrum recorded by means of an optical spectrum analyzer (ANDO AQ6317) is depicted in Fig. 1. The 3-dB and 10-dB bandwidths of the main emission line at $935 \mathrm{~nm}$ are $\sim 0.09 \mathrm{~nm}$ and $0.25 \mathrm{~nm}$, respectively.

Of course this is orders of magnitude larger than required $(<0.00047 \mathrm{~nm}$ or $160 \mathrm{MHz}$, respectively [11]). Therefore the technique of injection seeding [22, 23], was employed to

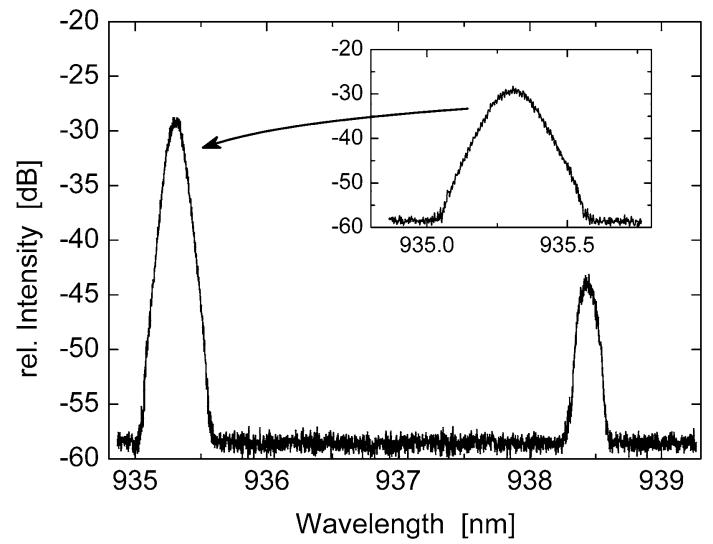

Fig. 1 Spectrum of the free-running Nd:YGG MOPA recorded by means of an optical spectrum analyzer at a resolution of $0.01 \mathrm{~nm}$. There are two emission lines visible at $935.31 \mathrm{~nm}$ and $938.43 \mathrm{~nm}$, respectively operate the MOPA laser in single-longitudinal-mode (SLM) operation.

\subsection{Injection seeding}

In order to seed the Nd:YGG MOPA and to consecutively switch its output between two wavelengths (i.e. on-line and off-line) from pulse to pulse, a concept was employed which has proven its reliability already during aircraft use [15].

This system (see Fig. 2) consists of four independent distributed-feedback (DFB) diode lasers of which in the context of this paper only three have been used. The diodes are operated at a current of $80 \mathrm{~mA}$, resulting in an output power of $35 \mathrm{~mW}$. The side-mode suppression is larger than $40 \mathrm{~dB}$. Changing the case temperature of the diodes between 18 and $28^{\circ} \mathrm{C}$ allows the (vacuum) wavelength to be set to an arbitrary value within the interval from 935.0 to $935.8 \mathrm{~nm}$. Fine tuning is then performed by changing the current. Each diode laser is collimated, fed through a 30-dB optical isolator to prevent back reflection, and coupled into a polarization-maintaining (PM) single-mode fiber. All subsequent fiber optic components which are in the light path used for seeding such as splitters, couplers, and switches of the seed laser system are likewise polarization maintaining.

In order to achieve high absolute frequency stability the first DFB laser (the "master") is frequency stabilized onto the strongest $\mathrm{H}_{2} \mathrm{O}$-absorption line $(935.6848 \mathrm{~nm})$ within the tuning range. This was done by using a multipass absorption cell with an optical path length of $36 \mathrm{~m}$ (Aerodyne AMAC36) and filled with $\sim 1 \mathrm{hPa}$ of water vapor. The frequency of the master laser is modulated at $4 \mathrm{kHz}$ and a standard lock-in technique is used to stabilize the DFB laser to the center of the $\mathrm{H}_{2} \mathrm{O}$-absorption line. The other DFB diode lasers are stabilized to a wavemeter (High Finesse WS7), which is continuously re-calibrated against the master (see
Fig. 2 Schematic set-up of the injection seeding system

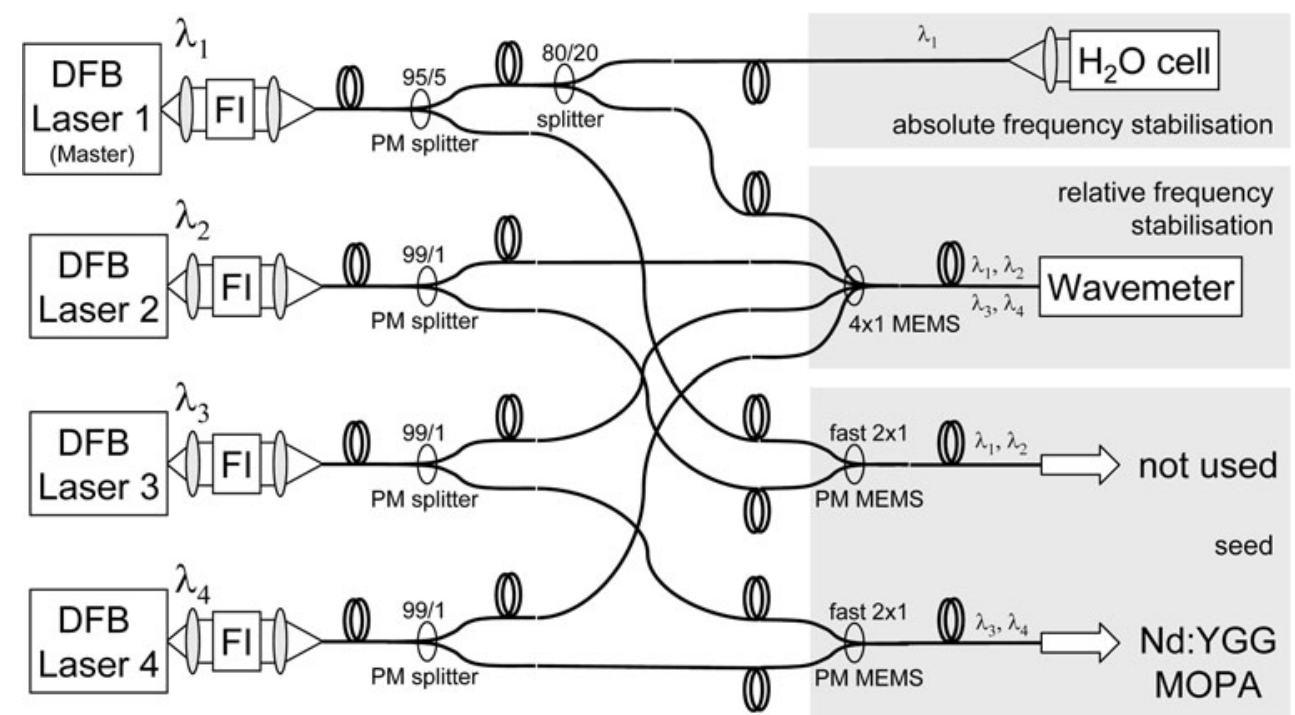


Fig. 2). The standard frequency deviation was previously determined to be $14.5 \mathrm{MHz}$ [15]. During the experiments described below, DFB laser \#3 and \#4 were used for injection seeding. Using a fast fiber-coupled micro-electromechanical switch (MEMS) the seed lasers are successively switched to the Nd:YGG oscillator cavity. It is foreseen to consecutively switch all four seed lasers to the oscillator using a cascade of three MEMS's but this was not yet implemented for the measurements described below. On the laser breadboard, the fiber output is finally collimated, propagated through another 30-dB Faraday-isolator to protect the seed laser system from depolarized laser pulses from the cavity, and geometrically matched to the oscillator cavity mode using a spherical telescope. Up to $\sim 5 \mathrm{~mW}$ of seed power were available in front of the Nd:YGG laser resonator.

The cavity length of the oscillator was matched to the seed wavelengths using the ramp-and-fire technique $[23,24]$. For this purpose, the transmitted seed light through the cavity was measured behind the outcoupling mirror using a photodiode. To protect the photodiode from optical damage as the laser pulses propagated along the same optical path as the seed laser signal the intensity was controlled by means of a half wave plate and a thin-film polarizer.

In single-frequency mode, and starting from an oscillator pulse energy of $4.3 \mathrm{~mJ}, 30.5 \mathrm{~mJ}$ could be generated from the MOPA system at gain maximum (935.31 nm) which corresponds to an overall optical efficiency of $3.4 \%$. In the experiments described below the yield was slightly lower ( $\sim 22-25 \mathrm{~mJ}$ ) which is contributed to minor misalignments of the pump diode coupling during transportation of the MOPA to the measurement site. By tuning the seed wavelength across the gain profile it was found that the range of single-frequency operation extended over $0.45 \mathrm{~nm}$ from $935.10 \mathrm{~nm}$ to $935.55 \mathrm{~nm}$. More details are given in Sect. 3.

The spectral width and frequency stability were measured in the companion paper [19] by means of a heterodyne technique [25]. The bandwidth was less than $15 \mathrm{MHz}$ (rms) and the frequency stability relative to the seed laser frequency was better than $3 \mathrm{MHz}$ (rms). These numbers are by far exceeding the requirements for water vapor DIAL.

\subsection{Detection system}

The receiving system consists of a $35-\mathrm{cm}$ Cassegrainian telescope. An interference filter with a bandwidth of $\sim 1 \mathrm{~nm}$ and a transmission of $>50 \%$ suppresses the solar background. The filter is heated to tune the center wavelength of its pass band to match the emission lines. As the detector an avalanche photo diode (APD) (Perkin Elmer C30955ETC) was employed which is temperature stabilized to $18^{\circ} \mathrm{C}$ to ensure a constant responsivity. The output signal from the
APD is digitized using a 14-bit digitizer at a $10 \mathrm{MHz}$ sampling rate resulting in a spatial resolution of $15 \mathrm{~m}$. The detector system is rigidly connected to the telescope frame. An overview on the main parameters of the receiving system is given in Table 2.

\subsection{Measurement set-up}

The overall measurement set-up is depicted in Fig. 3. Laser and receiving system were placed close to each other in the lab $\left(48^{\circ} 5^{\prime} 4^{\prime \prime} \mathrm{N}, 11^{\circ} 16^{\prime} 43^{\prime \prime} \mathrm{E}, 590 \mathrm{~m}\right.$ a.s.1.) but not rigidly connected. The PM-fiber from the seed laser system is attached to the oscillator. The MOPA laser is supplied from an electronics rack. It houses the pump diodes of the oscillator, the current drivers and cooling units of both oscillator and amplifier, the ramp-and-fire control electronics, and a PC since the laser is fully computer controlled. The trigger for the MEMS of the seed laser system is derived from the outgoing pulse and its operation is controlled by the data acquisition system. The backscatter signals for each switch position are individually stored.

Table 2 Summary of receiver properties

\begin{tabular}{ll}
\hline Parameter & Value \\
\hline Telescope diameter & $350 \mathrm{~mm}$ \\
Focal length & $5 \mathrm{~m}$ \\
Transmitter outcoupling & bistatic \\
Field-of-view & $\sim 1.5 \mathrm{mrad}$ \\
Interference filter bandwidth & $1 \mathrm{~nm}$ \\
Interference filter transmission & $>50 \%$ \\
Detector & APD \\
NEP & $8 \mathrm{fW} / \sqrt{ } \mathrm{Hz}$ \\
Analog/Digital converter & $14 \mathrm{bit}$ \\
Sampling Rate & $10 \mathrm{MHz}(15 \mathrm{~m})$ \\
\hline
\end{tabular}

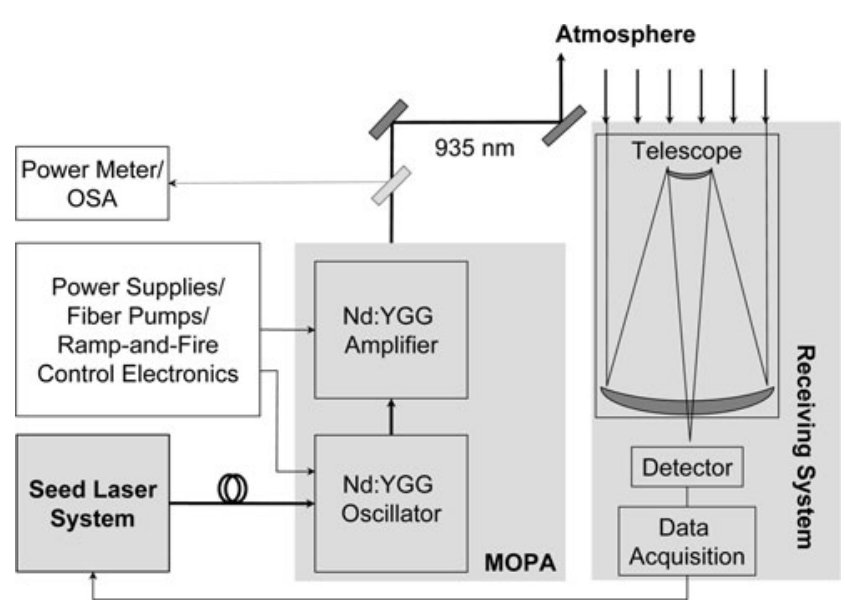

Fig. 3 Set-up of the entire lidar system 
By means of two steering mirrors the laser beam was directed out of the lab window at a slant angle of 7.5 degrees with respect to the horizon. This means that at a range of $12 \mathrm{~km}$ the sounding altitude equals $1570 \mathrm{~m}$. Therefore, the planetary boundary layer is more or less within the measurement range.

In order to characterize the laser properties the laser beam could be directed onto a power meter and also onto an integrating sphere that is fiber-coupled to an optical spectrum analyzer (ANDO AQ6317) to quantify the spectral properties.

\section{Measurements of the spectral purity}

Any DIAL measurement of trace species with a high accuracy in the atmosphere requires a high spectral purity $[8,21,26]$. Usually the spectral purity (SP) is defined as the ratio of the energy contained in a narrow spectral interval (typically the width of the absorption line) to the total energy. For a water vapor DIAL measurement through an atmosphere with a (2-way) optical thickness of 2 that has a systematic error of less than $1 \%$ the spectral purity must not fall below a value of $99.9 \%$ [9].

The accurate determination of a fraction of a percent of broadband light present in the laser beam makes it a challenging task. In order to investigate the seeding efficiency of the Nd:YGG laser as function of the seed wavelength we used the optical spectrum analyzer (OSA) and recorded the emission spectra of the injection-seeded Nd:YGG laser. First of all we recorded the spectra of the seeded laser over the range 934.5-939 $\mathrm{nm}$ that includes both emission lines. As expected, the emission at $938.4 \mathrm{~nm}$ vanishes the closer the seed wavelength coincides with the gain maximum at $935.3 \mathrm{~nm}$ (see Fig. 4). Since any spectral components that far away can be suppressed by appropriate filters, for water vapor DIAL measurements the spectral behavior in the vicinity of the main emission line is more important. Therefore spectra around $935.3 \mathrm{~nm}$ for various levels of seed power were recorded. Two of these spectra for different seed wavelengths (935.45 nm and $935.49 \mathrm{~nm}$, cf. Fig. 5) are depicted in Fig. 6. It is clearly seen that for the wavelength further in the wing of the gain curve modes in the vicinity of the gain center at $935.31 \mathrm{~nm}$ increase in intensity with decreasing seed power. For the wavelengths closer to the gain center only at seed powers as low as $\sim 10 \mu \mathrm{W}$ central modes become distinguishable from the background. In order to compare the behavior more quantitatively, the side-mode suppression ratio (SMSR) of the intensity at the gain center with respect to the intensity in the seeded mode was derived and is depicted in Fig. 7 . At the chosen resolution setting $(0.01 \mathrm{~nm})$ of the OSA side-mode suppression ratios of up to $\sim-30 \mathrm{~dB}$ could be resolved. The decrease of the SMSR appears to show a logarithmic dependence of the seed power, while as the curves are shifted toward lower seed powers the closer the seed wavelength is to the gain center. The total width of the wavelength range in which efficient injection seeding is maintained is therefore a matter of definition. Here, we state that efficient seeding requires that no modes at the gain center can be detected at the available seed powers $(\sim 4 \mathrm{~mW})$. Using this definition, the seeding range extends to $\sim 0.36 \mathrm{~nm}$ (see Fig. 5).

Measurements using the OSA, however, are not adequate to measure values of the spectral purity in the range of SP $=99.99 \%$ or higher. A convenient way to measure the spectral purity is the use of long-pass absorption cells [28-31]. This requires, however, that the transmission $\mathrm{T}$ of the cell is $\mathrm{T} \ll 1-\mathrm{SP}$. For the determination of the spectral purity of the Nd:YGG laser using absorption lines within the accessible wavelength range the cell has to be very long. It is therefore more convenient to measure the backscattered signal through an optically dense atmosphere. For the measurement conditions experienced at the time of the investigations $\left(T \approx 10^{\circ} \mathrm{C}, p \approx 950 \mathrm{hPa}\right.$; relative humidity $\approx 55 \%$ ) an absorption path of more than $5000 \mathrm{~m}$ (one-way) is needed to measure spectral purities of more than $99.9 \%$ at the line
Fig. 4 Spectra of the Nd:YGG laser between 934.5 and $939 \mathrm{~nm}$ when seeded at four different wavelengths. The arrows denote the spectral position of the respective seed wavelength. The seed power was $\sim 4 \mathrm{~mW}$ and the resolution of the spectrum analyzer was set to $0.1 \mathrm{~nm}$

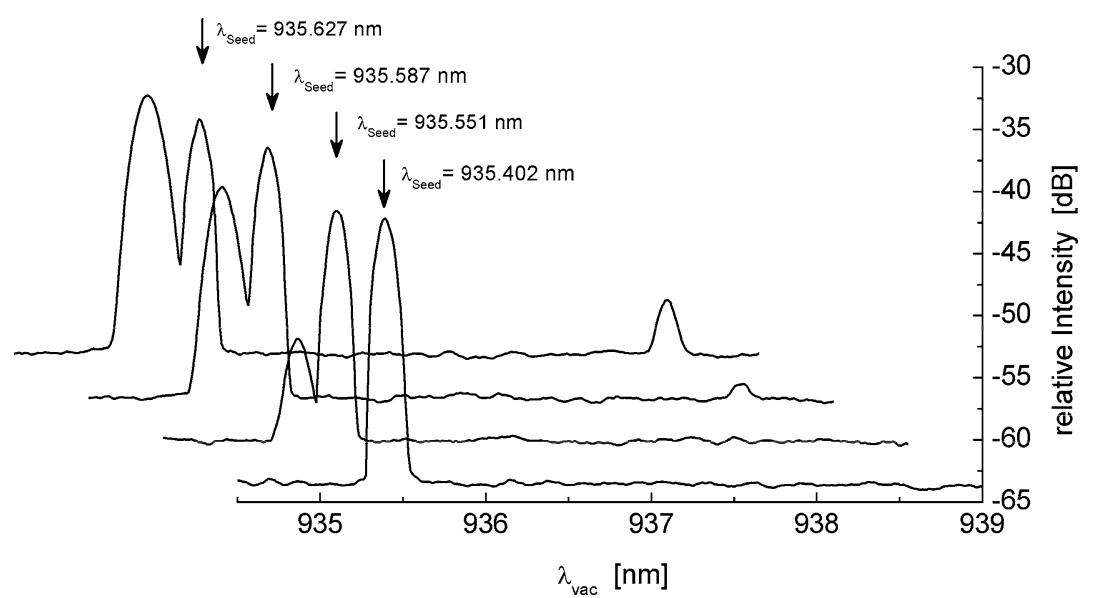




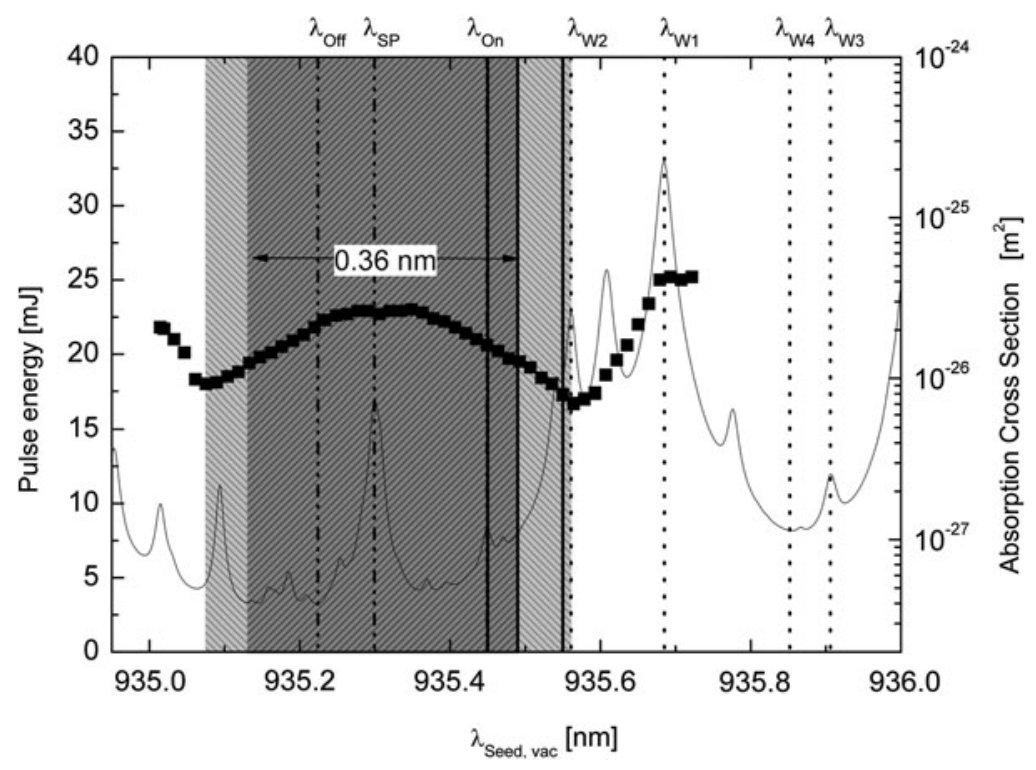

Fig. 5 Pulse energy of the Nd:YGG MOPA as function of the seed wavelength. In the darkly hatched wavelength range the laser is efficiently seeded. In the lightly hatched range seeding becomes less efficient and modes in the gain center start to oscillate. When the seed wavelength is too far off, the laser becomes totally unseeded and the energy equals the energy of the free-running system. Overlaid is the absorption cross section of water vapor (from HITRAN 2009 [27]) as well as the position of the selected WALES absorption lines $\left(\lambda_{W 1-4}\right.$; dotted lines), the position of the lines used to determine the seeding range (solid lines), and the measurement wavelengths used to determine the spectral purity and to perform the DIAL measurements $\left(\lambda_{\text {Off }}\right.$, $\lambda_{\mathrm{On}}, \lambda_{\mathrm{SP}} ;$ dash-dotted lines) at $\lambda=935.2992 \mathrm{~nm}$. More than $8000 \mathrm{~m}$ are required to detect spectral purities of $99.999 \%$. This is depicted in Fig. 8 where the optical transmission of this line (and two stronger lines at $935.5613 \mathrm{~nm}$ and $935.6848 \mathrm{~nm}$ ) is plotted versus the absorption length.

For the spectral purity measurements the set-up described in Sect. 2.4 was employed. We used the line at $935.2992 \mathrm{~nm}$ $\left(10691.765960 \mathrm{~cm}^{-1}\right)$ as the on-line wavelength using seed laser \#3 and, as the reference (off-line), a wavelength of $935.2241 \mathrm{~nm}$, which is considerably less absorbed by water vapor (see Fig. 5) using seed laser \#4. Both lasers were consecutively switched to the Nd:YGG oscillator.

The on-line $\left(P_{\text {on }}\right)$ and off-line $\left(P_{\text {off }}\right)$ backscatter signals were recorded and averaged over a time interval. Then, the spectral purity is calculated as

$\mathrm{SR} \geq \max \left[1-\left(\frac{P_{\mathrm{ref}}(r)}{P_{\mathrm{off}}(r)}\right)\right]$

Since $P_{\text {on }}$ is rapidly absorbed and eventually vanishes in the background noise of the detection system (see Fig. 9(a)) we chose as the reference level

$$
P_{\text {ref }}(r)=\max \left[P_{\text {on }}(r), \mathrm{DL}\right]
$$

where the detection limit DL was conservatively chosen to be the background level derived from the mean pretrigger signal and increased by one standard deviation. Apparently, the maximum spectral purity that can be derived is dependent on the signal-to-noise ratio and the dynamic range of the detection system (Fig. 9(b)). Since the near field signal must not be overridden in order not to introduce oscillations in the response of the avalanche photo diodes in clear atmosphere the maximum spectral purity that could be derived was on the order of $99.98 \%$.

However, a distant hard target that increases the off-line signal to almost full dynamic range can increase the maximum definable level of spectral purity. Due to the measurement geometry there was no stationary hard target, but in the measurement period clouds at the top of the planetary boundary layer occasionally entered the telescope's field-ofview acting as a suitable target. We chose an averaging period of ten minutes as a compromise between reducing the background noise and the smearing out of the hard target since the clouds are highly variable in time and backscatter properties. Care was taken that individual, overdriven backscatter signals from the clouds were not included in the evaluation process.

The measured spectral purity of the Nd:YGG MOPA as function of seed power is depicted in Fig. 10. At seed powers in the 3-4 $\mathrm{mW}$ range a degree of the spectral purity of as high as $99.996 \%$ was achieved. This is far above the requirements for water vapor DIAL. However, the numbers given are only a lower boundary for the spectral purity. For higher seed laser powers the on-line signal could not yet be distinguished from the detection limit and thus the spectral 

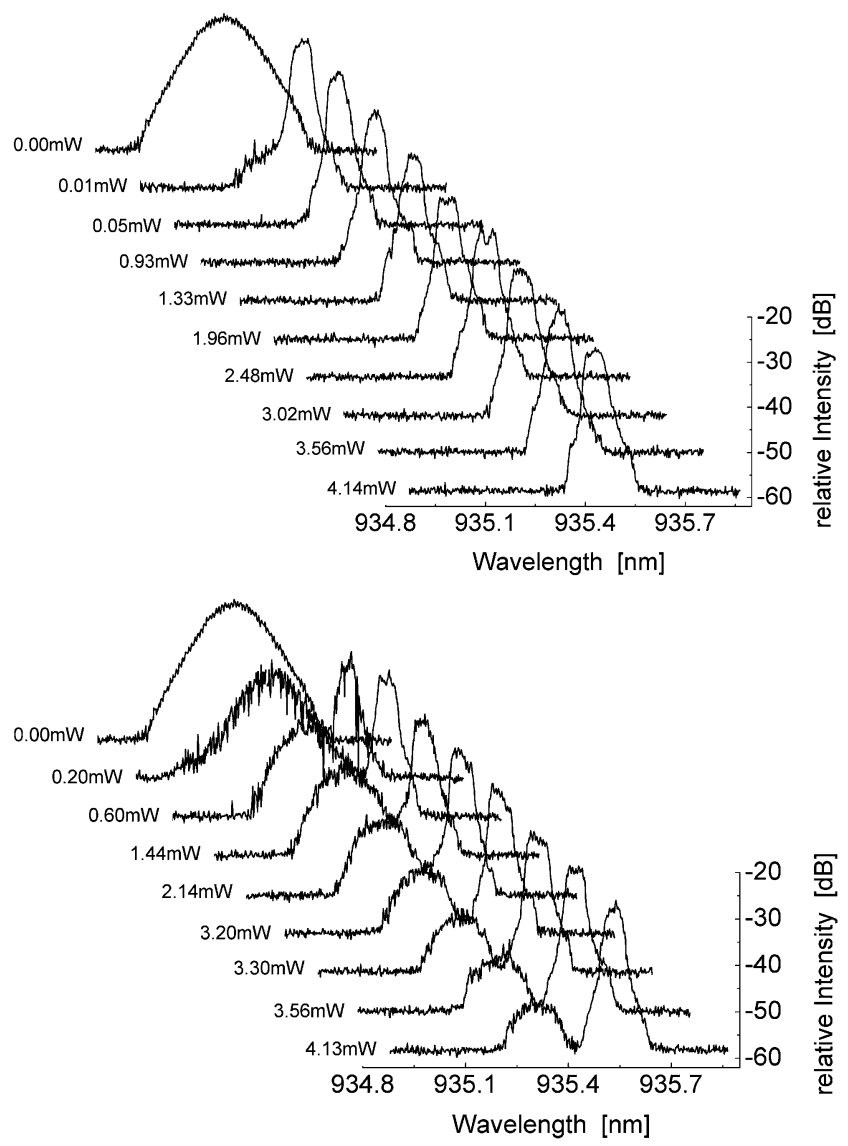

Fig. 6 Spectra of the Nd:YGG laser when seeded at two different wavelengths ( $935.45 \mathrm{~nm}$, upper graph; $935.49 \mathrm{~nm}$, lower graph) in the wing of the gain profile as function of the seed power

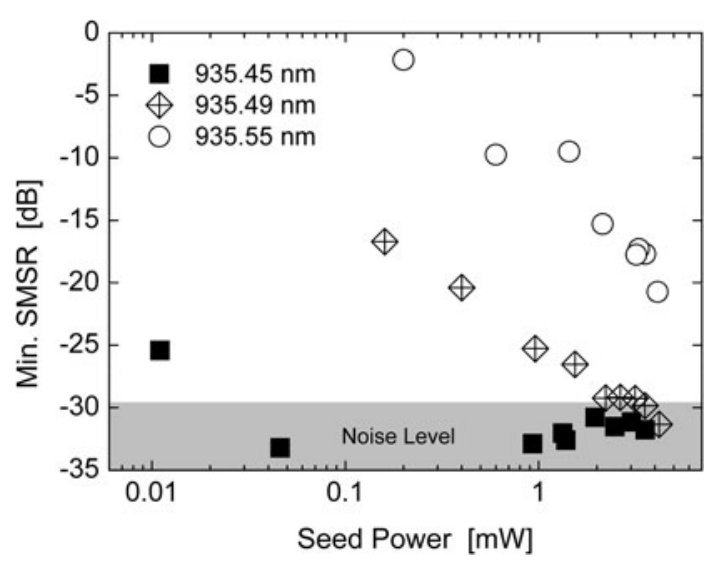

Fig. 7 Side-mode suppression ratio (SMSR) calculated from the measurements of the optical spectrum analyzer for three different seed wavelengths ( $935.45 \mathrm{~nm}, 935.49 \mathrm{~nm}$, and $935.55 \mathrm{~nm}$, respectively) in the wing of the Nd:YGG gain profile as function of seed power

purity is probably higher. For lower seed powers we noticed the very sporadic occurrence of badly seeded pulses. These pulses happen when electronic noise peaks occasionally became larger than the signal of the transmitted seed and trig-

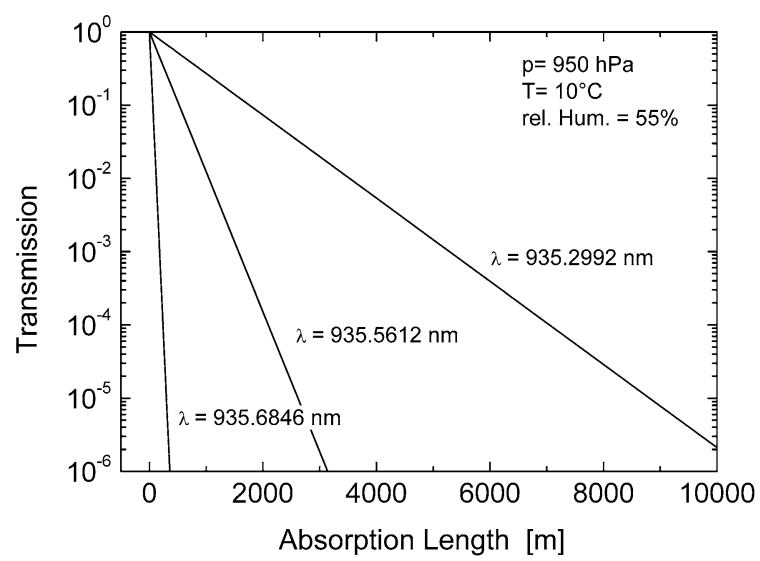

Fig. 8 Transmission through the atmosphere at three selected water vapor absorption lines around $935 \mathrm{~nm}$. At atmospheric conditions $p=950 \mathrm{hPa}, T=10^{\circ} \mathrm{C}$ and a relative humidity of $55 \%$ and at the strongest line within the tuning range of the Nd:YGG laser system $(935.2992 \mathrm{~nm})$ an absorption length of $>5000 \mathrm{~m}$ is required to achieve a one-way optical depth of $10^{-3}$

gered the Q-switch at a wrong point in time. If this happened only a few times within the averaging interval of ten minutes this would decrease the mean spectral purity significantly.

In either case, pulse energy, spatial as well as spectral properties of the Nd:YGG laser are well suited for DIAL measurements of water vapor.

\section{Water vapor DIAL measurements}

Several water vapor DIAL measurements were performed within the time period 3-20 April 2009. For these water vapor measurements the lines at $935.4491 \mathrm{~nm}$ and $935.2241 \mathrm{~nm}$ (see Fig. 5) were used for the on-line and offline wavelengths, respectively. For the retrieval of the water vapor profiles shown here the temperature- and pressuredependent line parameters from [27] were taken and applied within the simple DIAL equation. No further adjustments (such as the Rayleigh-Doppler correction) were applied.

The measurement period was characterized by fair weather. Typically, low pressure gradients prevailed over Central Europe and consequently the wind velocities were rather low.

The water vapor measurements could be compared with the operational radiosounding at Oberschleissheim, Germany $\left(48^{\circ} 14^{\prime} 43^{\prime \prime} \mathrm{N}, 11^{\circ} 33^{\prime} 21^{\prime \prime} \mathrm{E}, 489 \mathrm{~m}\right.$ a.s.1.), which is located at a distance of $27 \mathrm{~km}$ to the northeast of the lidar measurement site. At the same time, a continuous record from a standard weather station on site was available for in-situ data on the ground.

From the lidar data two showcase examples are presented here. The first example (Fig. 11) depicts a lidar measurement 


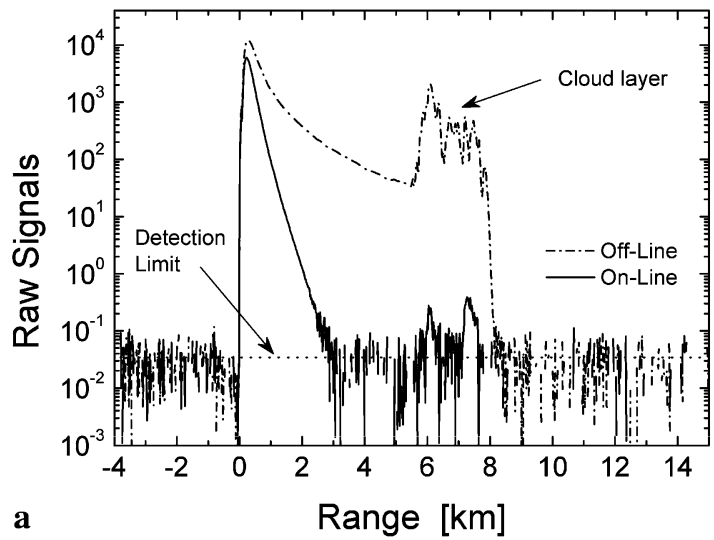

Fig. 9 (a) Raw signals of on-line and off-line signals exemplarily shown for a seed power of $0.8 \mathrm{~mW}$. The detection limit (mean value of the pretrigger signal enhanced by one standard deviation) is also shown. The cloud layer at $\sim 7 \mathrm{~km}$ range enhances the signal-to-noise

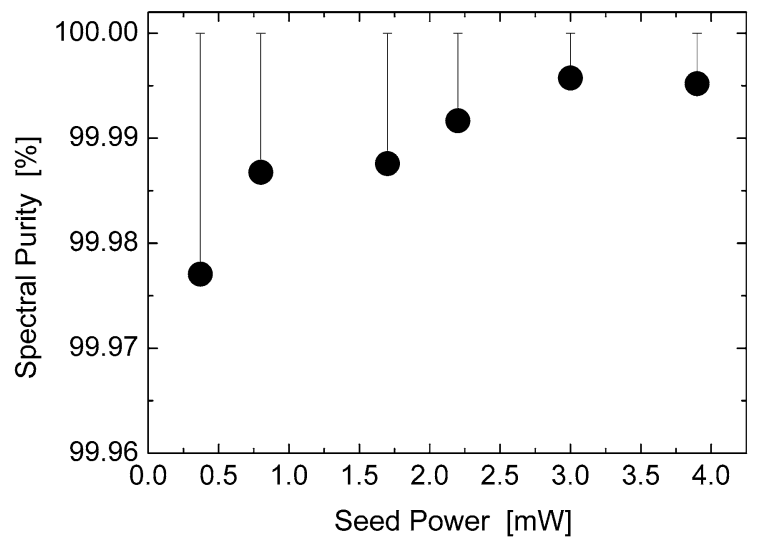

Fig. 10 Spectral purity of the Nd:YGG MOPA as function of the seed power in the range between 0.37 and $3.9 \mathrm{~mW}$. A spectral purity of as high as $99.996 \%$ was achieved

using the Nd:YGG MOPA system recorded during nighttime on 20 April 2009 between 20:36 and 22:04 UTC. The upper panel displays the attenuated backscatter ratio at the off-line wavelength in the altitude range from $0.8 \mathrm{~km}$ to $2.5 \mathrm{~km}$ a.s.l. Due to the measurement geometry this corresponds to a maximum range of $\sim 15 \mathrm{~km}$. The atmosphere appears to be rather clear with no pronounced aerosol structures. The water vapor mixing ratio is depicted in the lower panel. Due to the slanted measurement geometry the altitude resolution of the water vapor lidar data is as low as $30 \mathrm{~m}$. The temporal resolution was chosen to be 60 seconds. The data gap between 21:02 and 21:10 UTC was caused by the seed laser losing lock. The water vapor mixing ratio near the ground was on the order of $8 \mathrm{~g} / \mathrm{kg}$ decreasing to values around $3-5 \mathrm{~g} / \mathrm{kg}$ at altitudes of $1.4 \mathrm{~km}$. At this altitude a significant decrease in humidity takes place at $\sim 21$ UTC. An advection of dry air is also seen in the backscatter plot where around this time the backscatter ratio becomes reduced in

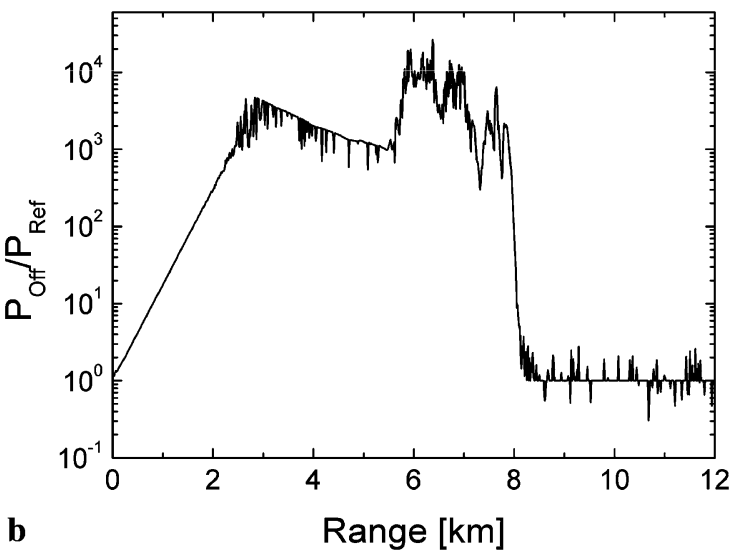

ratio and increases the value of the maximum spectral purity than can be detected. (b) For the raw signals given in (a) the ratio $P_{\text {off }} / P_{\text {Ref }}$ as function of range is displayed. From the maximum of this curve the spectral purity is derived according to (3.1)

this part of the atmosphere. Figure 12 shows the comparison of the radiosonde profile launched at 00 UTC in Oberschleissheim and the DIAL results. Also shown is the corresponding water vapor mixing ratio measured in-situ on the lidar site. In Fig. 12 two DIAL profiles are displayed which show the humidity profile before and after the dry layer has entered the field-of-view. Near the ground both lidar profiles show higher humidity than the radiosonde, but this is consistent with the in-situ measurement. While the profile that was recorded earlier shows a rather good agreement with the radiosonde, the later recording, although closer in time, shows slightly lower values. The likely reason for these deviations is a rather high wind shear as noticed from the radiosonde profile that complicates comparisons. It shows low north-easterly winds $(\sim 2.5 \mathrm{~m} / \mathrm{s})$ near the ground and much stronger winds $(\sim 12 \mathrm{~m} / \mathrm{s})$ turning to the east at an altitude of $\sim 1000 \mathrm{~m}$. On the lidar site the in-situ instruments also shows a very low north-easterly wind speed of less than $2 \mathrm{~m} / \mathrm{s}$.

The second example (Fig. 13) shows a daytime lidar measurement recorded on 8 April 2009 between 12:40 and 14:00 UTC using an identical resolution as for the above example. Faint clouds at the top of the planetary boundary layer (similar to those that acted as targets for the spectral purity measurements) appear at $\sim 13: 35$. The PBL height as derived from the radiosonde was $\sim 1650 \mathrm{~m}$ a.s.1., which is consistent with the lidar data. Again, the water vapor mixing ratio is depicted in the lower panel. The planetary boundary layer appears to be well mixed. Compared to Fig. 11 the water vapor mixing ratio near the ground was lower $(\sim 5.5 \mathrm{~g} / \mathrm{kg})$ on this day. This is also the reason why the range where the humidity could be retrieved with a low statistical error extends to slightly higher altitudes.

Figure 14 shows the comparison of the radiosonde profile launched at 12 UTC in Oberschleissheim to the average 


\section{0-04-2009}
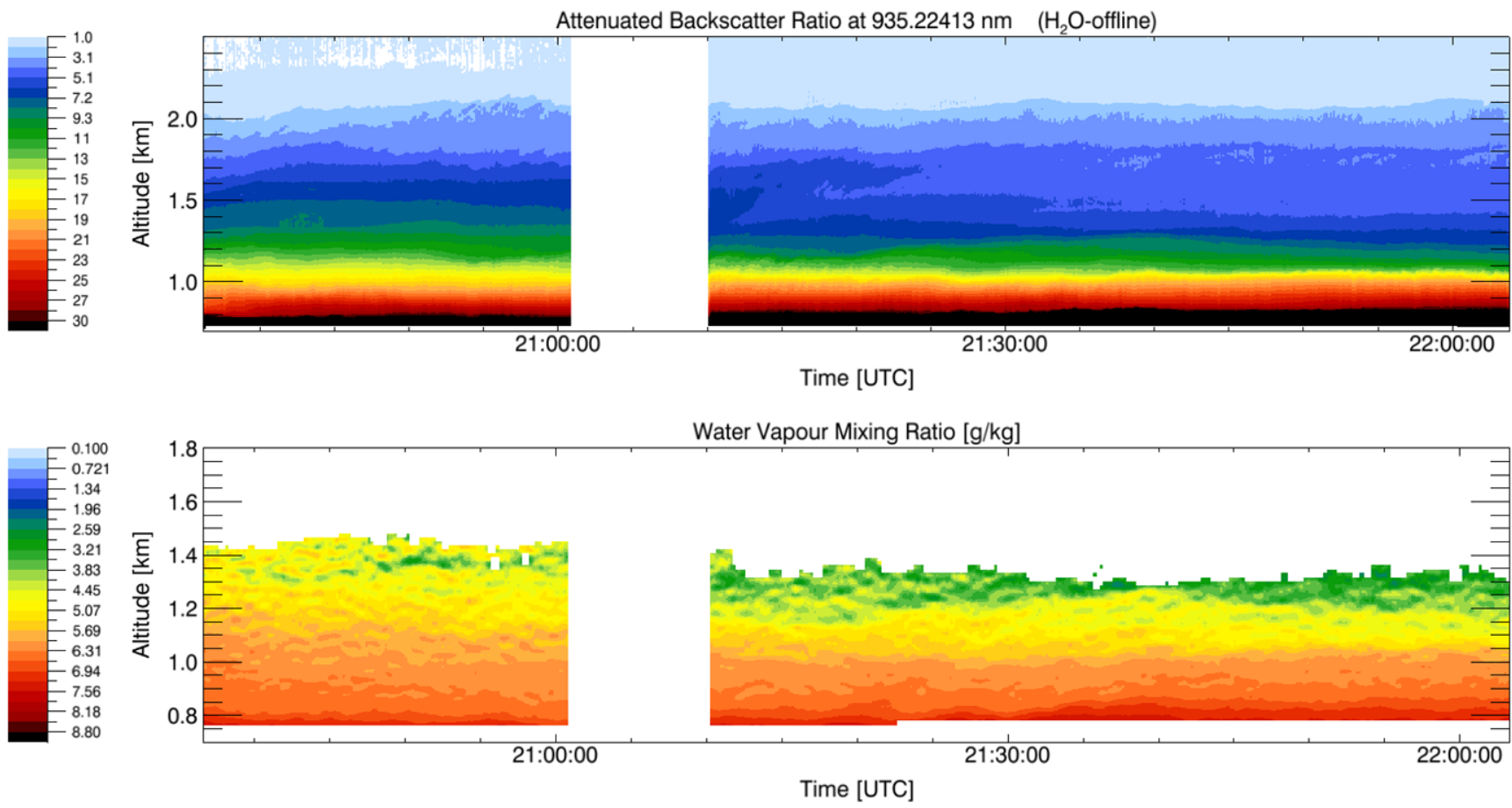

Fig. 11 Lidar measurement using the Nd:YGG MOPA system recorded on 20 April 2009 between 20:36 and 22:04 UTC. Upper panel: Attenuated backscatter ratio in the altitude range from $0.8 \mathrm{~km}$

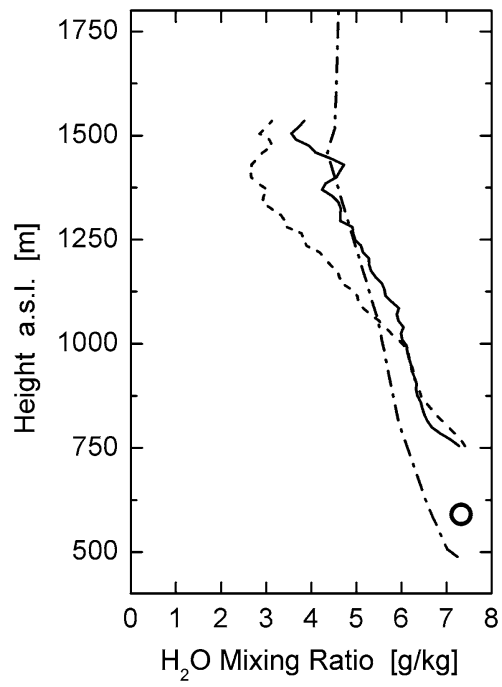

Fig. 12 Comparison of two DIAL water vapor profiles recorded on 20 Apr 2009 at 20:48 UTC (solid line) and at 21:30 UTC (dashed line) to the profile of the Oberschleissheim radiosonde launched on $21 \mathrm{Apr}$ 2009 at 00 UTC (dash-dotted line). The corresponding water vapor mixing ratio measured in-situ on the lidar site is given by the circle

DIAL water vapor profile recorded between 12:40 and 14:00 UTC. The corresponding water vapor mixing ratio measured in-situ on the lidar site is also given. Here, it facilitates com- to $2.5 \mathrm{~km}$ a.s.1. Lower panel: Water vapor mixing ratio (in $\mathrm{g} / \mathrm{kg}$ ) in the altitude range from $0.8 \mathrm{~km}$ to $1.8 \mathrm{~km}$ a.s.l. Due to the slanted measurement geometry the altitude resolution is as low as $30 \mathrm{~m}$

parisons that the well-mixed boundary layer shows a very homogeneous water vapor profile. Low westerly winds with a wind speed of below $2 \mathrm{~m} / \mathrm{s}$ prevailed without showing significant wind shear throughout the boundary layer in the radiosonde data. Despite a difference of $\sim 1 \mathrm{~h}$ and $27 \mathrm{~km}$ in time and distance, respectively, the agreement between lidar and radiosonde profile is excellent particularly above altitudes where ground effects become negligible. These measurements impressively demonstrate the capability of the Nd:YGG laser for water vapor DIAL measurements.

\section{Summary}

In summary, we presented to the best of our knowledge for the first time water vapor differential absorption lidar measurement using a directly-diode pumped solid-state laser at $\sim 935 \mathrm{~nm}$. The results show good agreement to in-situ profiles recorded by means of radiosondes.

Since, next to energy and spatial beam profile, the spectral requirements and particularly the spectral purity are of utmost importance to achieve a high accuracy of the lidar measurement those properties have been investigated in detail. Values of the spectral purity of better than $99.996 \%$ were achieved using the technique of injection seeding. These values that exceed the requirements for spaceborne 
08-04-2009
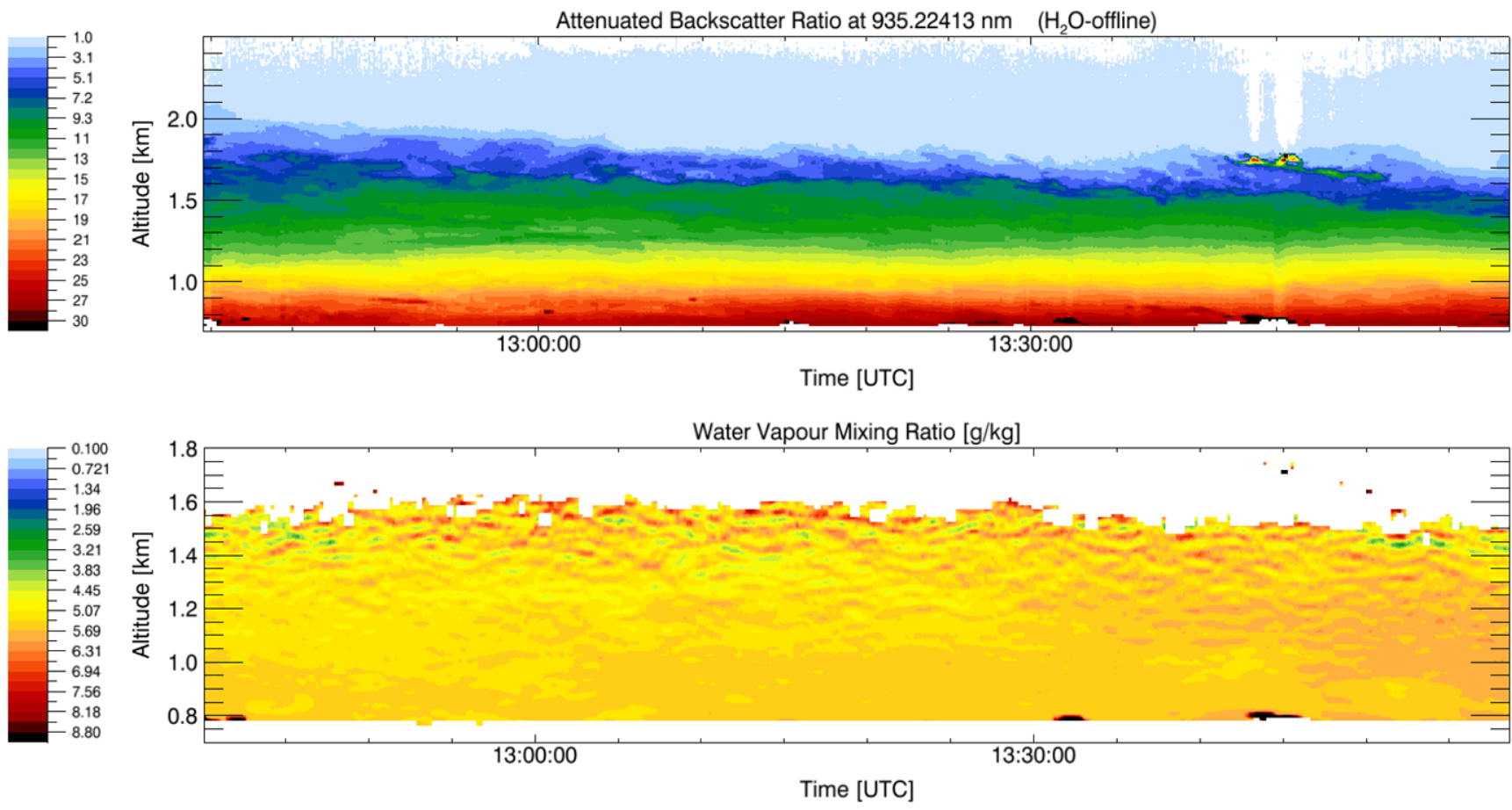

Fig. 13 Lidar measurement using the Nd:YGG MOPA system recorded on 8 April 2009 between 12:40 and 14:00 UTC. Upper panel: Attenuated backscatter ratio in the altitude range from $0.8 \mathrm{~km}$

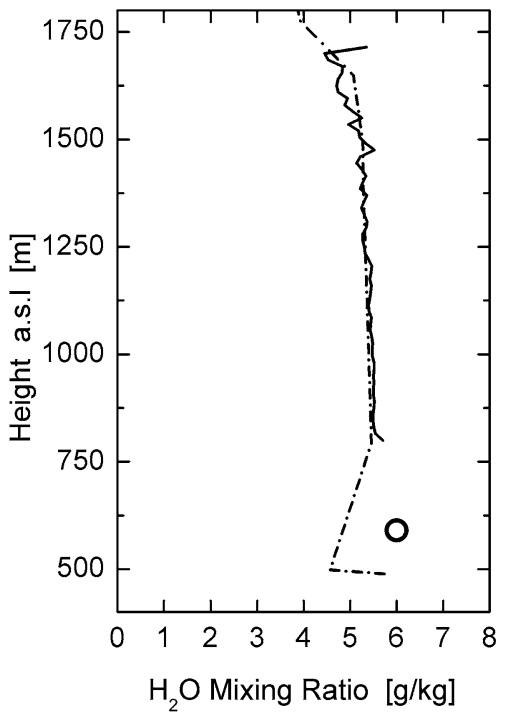

Fig. 14 Comparison of the DIAL water vapor profile recorded on 8 Apr 2009 (solid line) to the profile of the Oberschleissheim radiosonde launched at 12 UTC (dash-dotted line). The corresponding water vapor mixing ratio measured in-situ on the lidar site is given by the circle

H2O DIAL by far were confirmed by long-pass absorption measurement in the atmosphere. Such high degrees of spectral purity would be even appropriate for lidar measurements of carbon dioxide or methane at $\sim 1.6 \mu \mathrm{m}$ which are more to $2.5 \mathrm{~km}$ a.s.1. Lower panel: Water vapor mixing ratio (in $\mathrm{g} / \mathrm{kg}$ ) in the altitude range from $0.8 \mathrm{~km}$ to $1.8 \mathrm{~km}$ a.s.l.

demanding than for water vapor [32]. For that wavelength range lasers with similar host materials but using erbium instead of neodymium as the dopant are interesting candidates [33].

The only drawback was the fact that the strongest absorption lines $(935.6864 \mathrm{~nm})$ and thus part of the wavelengths proposed for a spaceborne H2O DIAL mission [10] could not be reached using Nd:YGG as the laser material since the gain spectrum restricted single-frequency operation with high spectral purity from $935.13 \mathrm{~nm}$ to $935.49 \mathrm{~nm}$. However, efforts are underway to investigate the crystal system $\mathrm{Nd}: \mathrm{Y}_{x} \mathrm{Lu}_{3-x} \mathrm{Ga}_{5} \mathrm{O}_{12}$. Preliminary investigations already revealed that the emission spectra of this crystal system can be compositionally tuned to cover the whole wavelength region of interest [34, 35].

In conclusion, this class of directly diode-pumped solid state lasers are now raised to a technology readiness level that make them competitive to current Ti:Sapphire laser or OPO systems as highly efficient transmitter for future spaceborne water vapor DIAL transmitters. The feasibility of a spaceborne mission for active remote sensing of atmospheric water vapor thus has significantly been promoted.

Acknowledgements This project was funded by DLR space agency as representative for BMWi under contract number: FKZ 50 EE 0714. We would like to thank Alexander Wissel, Martin Wirth, and Michael Esselborn for helping setting up the measurement equipment and their 
assistance during the measurements. We also acknowledge DLR flight department for providing adequate lab space.

Open Access This article is distributed under the terms of the Creative Commons Attribution Noncommercial License which permits any noncommercial use, distribution, and reproduction in any medium, provided the original author(s) and source are credited.

\section{References}

1. Climate Change, The physical science basis, in Contribution of Working Group I to the Fourth Assessment Report of the Intergovernmental Panel on Climate Change, ed. by S. Solomon, D. Qin, M. Manning, Z. Chen, M. Marquis, K.B. Averyt, M. Tignor, H.L. Miller (Cambridge University Press, Cambridge, 2007),

2. L. Bengtsson, K.I. Hodges, S. Hagemann, Tellus 56A, 202 (2004)

3. E. Andersson, E. Hòlm, P. Bauer, A. Beljaars, G.A. Kelly, A.P. McNally, A.J. Simmons, J.-N. Thépaut, A.M. Tompkins, Q. J. R. Meteorol. Soc. 133, 1473 (2007)

4. C. Keil, A. Röpnack, G.C. Craig, U. Schumann, Geophys. Res. Lett. 35, L09812 (2008). doi:10.1029/2008GL033657

5. WMO Space Programme SP-7. "The space-based global observing system in 2010 (GOS-2010)" WMO/TD-No. 1513 (2010)

6. WCRP, 2000: Assessment of upper tropospheric and stratospheric water vapour. SPARC Rep. 2, WCRP 113, WMO Tech. Doc. 1043, $312 \mathrm{pp}$.

7. E. Gérard, D.G.H. Tan, L. Garand, V. Wulfmeyer, G. Ehret, P. Di Girolamo, Bull. Am. Meteorol. Soc. 85, 237 (2004)

8. S. Ismail, E.V. Browell, Appl. Opt. 28, 3603 (1989)

9. E.V. Browell, S. Ismail, W.B. Grant, Appl. Phys. B 67, 399 (1998)

10. ESA (European Space Agency), 2001: WALES-WAter Vapour Lidar Experiment in Space. The Five Candidate Earth Explorer Core Missions, European Space Agency, Report for Assessment, ESA SP-1257(2), September 2001

11. ESA, Report for Mission Selection: WALES-Water Vapour Lidar Experiment in Space. ESA SP 1279 (3). ISBN 92-9092-962-6 (2004)

12. A. Hélière, J.-L. Bézy, P. Bensi, P. Ingmann, Proc. SPIE Int. Soc. Opt. Eng. 4881, 24 (2003)

13. P. Di Girolamo, A. Behrendt, C. Kiemle, V. Wulfmeyer, H. Bauer, D. Summa, A. Dörnbrack, G. Ehret, Remote Sens. Environ. 112, $1552(2008)$

14. A.S. Moore Jr., K.E. Brown, W.M. Hall, J.C. Barnes, W.C. Edwards, L.B. Petway, A.D. Little, W.S. Luck Jr., I.W. Jones, C.W. Antill Jr., E.V. Browell, S. Ismail, in Advances in Atmospheric Remote Sensing with Lidar, ed. by A. Ansmann, R. Neuber, P. Rairoux, U. Wandinger (Springer, Berlin, 1997), pp. 281-288
15. M. Wirth, A. Fix, P. Mahnke, H. Schwarzer, F. Schrandt, G. Ehret, Appl. Phys. B 96, 201 (2009)

16. B.M. Walsh, N.P. Barnes, R.L. Hutcheson, R.W. Equall, B. Di Bartolo, J. Opt. Soc. Am. B 15, 2794 (1998)

17. N.P. Barnes, Opt. Mat. 27, 1653 (2005)

18. ESA Earth, Science Advisory Committee, private communication, April 2006

19. J. Löhring, A. Meissner, D. Hoffmann, A. Fix, G. Ehret, M. Alpers, Diode-pumped single-frequency-Nd:YGG-MOPA for water vapor DIAL measurements: Design, setup and performance. Appl. Phys. B (2010, to be published). doi:10.1007/ s00340-010-4314-1

20. P. Russbueldt, T. Mans, G. Rotarius, J. Weitenberg, H.D. Hoffmann, R. Poprawe, Opt. Express 17, 12230 (2009)

21. V. Wulfmeyer, J. Bösenberg, Appl. Opt. 37, 3825 (1998)

22. L.A. Rahn, Appl. Opt. 24, 940 (1985)

23. S.W. Henderson, E.H. Yuen, E.S. Fry, Opt. Lett. 11, 715 (1986)

24. K. Nicklaus, V. Morasch, M. Hoefer, J. Luttmann, M. Vierkötter, M. Ostermeyer, J. Höffner, C. Lemmerz, D. Hoffmann, Proc. SPIE 6451, 64511L (2007)

25. T. Schröder, C. Lemmerz, O. Reitebuch, M. Wirth, C. Wührer, R. Treichel, Appl. Phys. B 87, 437 (2007)

26. J. Bösenberg, Appl. Opt. 37, 3845 (1998)

27. L.S. Rothman, I.E. Gordon, A. Barbe, D.C. Benner, P.F. Bernath, M. Birk, L.R. Brown, V. Boudon, J.-P. Champion, K.V. Chance, L.H. Coudert, V. Dana, M.V. Devi, S. Fally, J.-M. Flaud, R.R. Gamache, A. Goldman, D. Jacquemart, N. Lacome, J.-Y. Mandin, S.T. Massie, S. Mikhailenko, A. Nikitin, J. Orphal, V. Perevalov, A. Perrin, C.P. Rinsland, M. Šimečková, M.A.H. Smith, S. Tashkun, J. Tennyson, R.A. Toth, A.C. Vandaele, J. Van der Auwera, J. Quantum Spectrosc. Radiat. Transfer 110, 533 (2009)

28. G. Poberaj, A. Fix, A. Assion, M. Wirth, C. Kiemle, G. Ehret, Appl. Phys. B 75, 165 (2002)

29. G.K. Schwemmer, M. Dombrowski, C.L. Korb, J. Milrod, H. Walden, R.H. Kagann, Rev. Sci. Instrum. 58, 2226 (1987)

30. P. Ponsardin, N.S. Higdon, B.E. Grossmann, E.V. Browell, Appl. Opt. 33, 6439 (1994)

31. P. Mahnke, H.H. Klingenberg, A. Fix, M. Wirth, Appl. Phys. B 89, 1 (2007)

32. G. Ehret, C. Kiemle, M. Wirth, A. Amediek, A. Fix, S. Houweling, Appl. Phys. B 90, 593 (2008)

33. H. Stange, K. Petermann, G. Huber, E.W. Duczynski, Appl. Phys. B 49, 269 (1989)

34. R. Treichel, C. Czeranowsky, B. Ileri, K. Petermann, G. Huber, in Proceedings of the 5th International Conference on Space Optics (ICSO 2004), ESA SP-554, ed. by B. Warmbein (ESA Publications Division, Noordwijk, 2004), pp. 639-642. ISBN92-9092-865-4

35. J. Löhring (Fraunhofer ILT, Aachen) and L. Ackermann (FEE $\mathrm{GmbH}$, Idar-Oberstein), private communication (2010) 